\title{
Portosystemic Encephalopathy without Liver Cirrhosis Masquerading as Depression
}

\author{
Takanori Asakura ${ }^{1}$, Nobutake Ito $^{2}$, Takahiro Sohma ${ }^{2}$ and Nobuaki Mori ${ }^{1}$
}

\begin{abstract}
A 69-year-old woman was hospitalized due to progressive lethargy with hyperammonemia. Five months before the current admission, she was diagnosed with depression based on her low level of daily physical activity and thus began taking antidepressants. Abdominal computed tomography revealed a portosystemic shunt running between the left renal vein and inferior mesenteric vein. Balloon-occluded retrograde transvenous obliteration of the shunt vessel was performed, and the patient showed a remarkable clinical improvement. The possibility of a portosystemic shunt should be considered in the presence or absence of underlying liver disease and the ammonia level should be measured before diagnosing depression, as portosystemic encephalopathy may be reversible with interventional radiology treatment.
\end{abstract}

Key words: depression, portosystemic encephalopathy, portosystemic shunt

(Intern Med 54: 1619-1622, 2015)

(DOI: 10.2169/internalmedicine.54.3800)

\section{Introduction}

Portosystemic shunts primarily occur in cases of portal hypertension with liver cirrhosis and subsequently lead to portosystemic encephalopathy, a reversible syndrome characterized by an impaired brain function. Although portosystemic encephalopathy has also been reported to develop in patients with portosystemic shunts without liver cirrhosis, many of these patients have been misdiagnosed as having dementia or other psychological disorders and the condition represents a diagnostic challenge for clinicians. We herein describe a case of portosystemic encephalopathy in a patient without liver cirrhosis who was misdiagnosed to have depression and showed a remarkable clinical improvement after undergoing balloon-occluded retrograde transvenous obliteration (B-RTO).

\section{Case Report}

A 69-year-old woman presented to our hospital with progressive lethargy. An altered mental status had been recognized six months previously. Five months before the current admission, she was diagnosed with depression by a primary care physician due to her low level of daily physical activity and began taking antidepressants. She also took glycyrrhizin (100 mg per day) for allergic dermatitis. She had no personal or family history of liver disease, such as acute or chronic viral hepatitis and autoimmune disease, abdominal injury, surgery or blood transfusions and did not drink alcohol or use illicit drugs. Her Glasgow Coma Scale was 12 (E2V4M6). Her blood pressure was $158 / 94 \mathrm{mmHg}$, her body temperature was $39.1^{\circ} \mathrm{C}$, her pulse rate was 131 beats per minute, her respiratory rate was 16 breaths per minute and her oxygen saturation was $98 \%$ on ambient air. In addition, her weight was $56.5 \mathrm{~kg}$, her height was $149.8 \mathrm{~cm}$ and her body mass index was $25.2 \mathrm{~kg} / \mathrm{m}^{2}$. A physical examination showed asterixis, although no other signs indicative of liver cirrhosis, such as jaundice, spider angioma, palmar erythema, an increased liver size or ascites, were noted. Furthermore, there were no signs of meningeal irritation, and the physical and neurological findings were unremarkable. The results of laboratory tests are shown in Table. The findings of a cerebral fluid analysis were normal, and abdominal ultrasonography showed only fatty liver, with no evidence of an intrahepatic shunt or splenomegaly. Meanwhile, an ab-

${ }^{1}$ Department of General Internal Medicine, National Hospital Organization Tokyo Medical Center, Japan and ${ }^{2}$ Department of Radiology, National Hospital Organization Tokyo Medical Center, Japan

Received for publication August 1, 2014; Accepted for publication November 4, 2014

Correspondence to Dr. Takanori Asakura, takanori.asakura@gmail.com 
Table. Laboratory Data on Admission.

\begin{tabular}{|c|c|c|c|c|c|c|c|c|}
\hline Hematology & & & Biochemistry & & & Serology & & \\
\hline WBC & 13,800 & $/ \mu \mathrm{L}$ & TP & 8.2 & $\mathrm{~g} / \mathrm{dL}$ & CRP & 2.4 & $\mathrm{mg} / \mathrm{dL}$ \\
\hline $\mathrm{Hb}$ & 15.8 & $\mathrm{~g} / \mathrm{dL}$ & Alb & 3.8 & $\mathrm{~g} / \mathrm{dL}$ & $\operatorname{IgG}$ & 1,786 & $\mathrm{mg} / \mathrm{dL}$ \\
\hline $\mathrm{Ht}$ & 45.5 & $\%$ & Uric acid & 10.7 & $\mathrm{mg} / \mathrm{dL}$ & $\operatorname{IgA}$ & 256 & $\mathrm{mg} / \mathrm{dL}$ \\
\hline \multirow[t]{2}{*}{ Plt } & $29.4 \times 10^{4}$ & $/ \mu \mathrm{L}$ & BUN & 16.2 & $\mathrm{mg} / \mathrm{dL}$ & $\operatorname{IgM}$ & 345 & $\mathrm{mg} / \mathrm{dL}$ \\
\hline & & & $\mathrm{Cr}$ & 1.04 & $\mathrm{mg} / \mathrm{dL}$ & HBs-Ag & $(-)$ & $0.2 \mathrm{IU} / \mathrm{mL}$ CLIA \\
\hline Coagulation & & & T-Bil & 5.22 & $\mathrm{mg} / \mathrm{dL}$ & Anti-HBs & $(+)$ & 18.3 index CLIA \\
\hline APTT & 30.1 & $\mathrm{~s}$ & D-Bil & 2.86 & $\mathrm{mg} / \mathrm{dL}$ & Anti-HBc & $(+)$ & 5.7 index CLIA \\
\hline $\mathrm{PT} \%$ & 74.2 & $\%$ & LDH & 354 & $\mathrm{IU} / \mathrm{L}$ & HBe-Ag & $(-)$ & 0.1 index CLIA \\
\hline PT & 1.13 & & AST & 77 & $\mathrm{IU} / \mathrm{L}$ & Anti-HBe & $(-)$ & 15.1 index CLIA \\
\hline \multirow[t]{9}{*}{ FIB } & 340 & $\mathrm{mg} / \mathrm{dL}$ & ALT & 40 & $\mathrm{IU} / \mathrm{L}$ & HBV-DNA & $(-)$ & undetectable \\
\hline & & & ALP & 361 & $\mathrm{IU} / \mathrm{L}$ & $\mathrm{HCV}-\mathrm{Ab}$ & $(-)$ & $<1.0 \quad \log \mathrm{U} / \mathrm{mL}$ \\
\hline & & & $\gamma$-GTP & 67 & $\mathrm{IU} / \mathrm{L}$ & ANA & $(+)$ & $1: 160$ titer Speckled, Homogeneous \\
\hline & & & $\mathrm{ChE}$ & 163 & $\mathrm{U} / \mathrm{L}$ & AMA2 & $(-)$ & \\
\hline & & & $\mathrm{Na}$ & 150 & $\mathrm{mEq} / \mathrm{L}$ & ASMA & $(-)$ & \\
\hline & & & $\mathrm{K}$ & 2.3 & $\mathrm{mEq} / \mathrm{L}$ & & & \\
\hline & & & $\mathrm{Cl}$ & 100 & $\mathrm{mEq} / \mathrm{L}$ & & & \\
\hline & & & Ferritin & 118 & $\mathrm{ng} / \mathrm{mL}$ & & & \\
\hline & & & Ammonia & 228 & $\mu \mathrm{g} / \mathrm{dL}$ & & & \\
\hline
\end{tabular}

$\gamma$-GTP: $\gamma$-glutamyltransferase, Alb: albumin, ALP: alkaline phosphatase, ALT: alanine aminotransferase, AMA: anti-mitochondrial antibody, ANA: anti-nuclear antibody, APTT: activated partial thromboplastin time, ASMA: anti-smooth muscle antibody, AST: aspartate aminotransferase, BUN: blood urea nitrogen, ChE: cholinesterase, Cr: creatinine, CRP: C reactive protein, D-Bil: direct bilirubin, FIB: fibrinogen, Hb: hemoglobin, Ht: hematocrit, Ig: immunoglobulin, LDH: lactate dehydrogenase, Plt: platelet, PT: prothrombin time, PT-INR: prothrombin-international normalized ratio, T-Bil: total bilirubin, TP: total protein, WBC: white blood cell

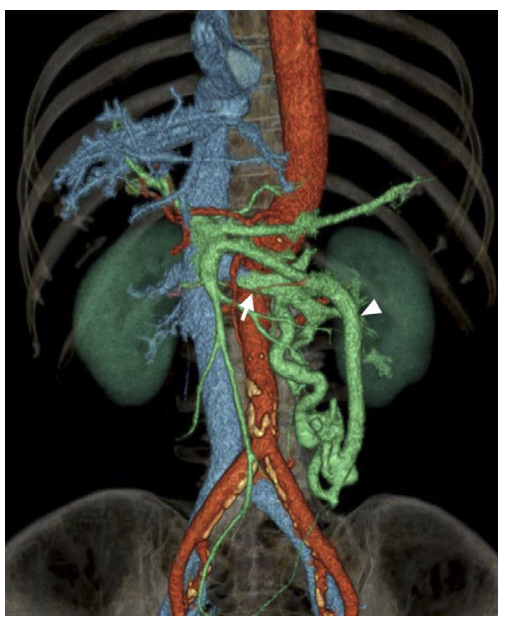

Figure 1. An abdominal computed tomography scan with contrast enhancement revealed a portosystemic shunt running between the left renal vein (arrow) and inferior mesenteric vein (arrowhead).

dominal computed tomography scan with contrast enhancement demonstrated a portosystemic shunt running between the left renal vein and inferior mesenteric vein (Fig. 1).

After admission, intravenous branched-chain amino acid and intracolonic lactulose were administered for portosystemic encephalopathy in addition to antibiotics, and fluid resuscitation was performed. The liver function abnormalities and hyperbilirubinemia improved; however, there was little improvement in the patient's consciousness or serum ammonia level. Urine and blood cultures were negative. On day 7 , B-RTO was performed to treat the shunt vessel and medically refractory portosystemic encephalopathy (Fig. 2) B-
RTO was selected because it is an effective and minimally invasive technique. There were no procedure-related complications, such as infection, contrast-induced nephropathy, hematoma formation, pulmonary embolism, edema or hemolysis, and esophagogastroduodenoscopy showed no gastroesophageal varices. The treatment resulted in a notable clinical improvement, and the serum ammonia level normalized. There were no portal hypertensive complications throughout the eight-month follow-up period, and the patient remained free from hyperammonemia and symptoms of depression without treatment with antidepressants.

\section{Discussion}

The present case of portosystemic encephalopathy involved a patient without liver cirrhosis who was currently receiving treatment for depression. In previous reports, the etiology of portosystemic encephalopathy without liver cirrhosis included congenital abnormalities in the intrahepatic vascular system, degeneration of the hepatic parenchyma and anastomosing vascularization after abdominal surgery, liver biopsy or trauma (1). In the current case, while the serum levels of hyaluronic acid and the type IV collagen $7 \mathrm{~s}$ domain, a marker of hepatic fibrosis, were elevated, the levels of serum albumin, platelets and ferritin were normal and there were no signs of portal hypertension, such as splenomegaly or gastroesophageal varices. A liver biopsy was not performed to clarify the underlying liver disease because the patient did not consent to this procedure. Although she may have had a chronic liver disease, such as nonalcoholic fatty liver, her laboratory and imaging data indicated a preserved liver function, with the exception of ele- 

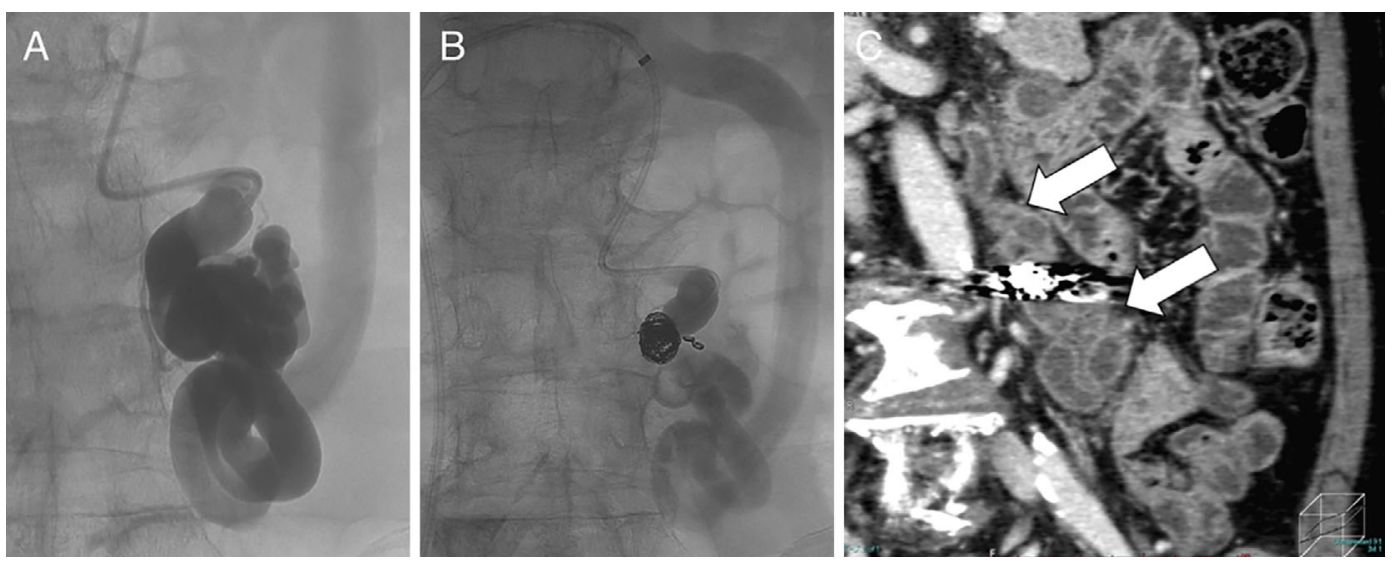

Figure 2. A 5-Fr balloon catheter was advanced into the portogonadal shunt through the right femoral vein. After confirming nearly complete stagnation on balloon occlusion venography (A), the shunt was filled with a sclerosant (5\% ethanolamine oleate iopamidol). Although the balloon was inflated and remained in place for $\mathbf{2 0}$ minutes after the injection, complete hemostasis was not achieved. Therefore, the balloon was left in place overnight to enhance coil embolization of the shunt (B). A computed tomography scan obtained one week later revealed complete occlusion of the portogonadal shunt $(\mathrm{C}$, the arrows indicate thrombi in the shunting vein).

vated levels of hepatic fibrosis markers. Akahoshi et al. reported a case of pathological fibrosis of the liver with portosystemic encephalopathy in a patient without cirrhosis due to the presence of a congenital extrahepatic portosystemic shunt (2). However, follow-up studies are needed to evaluate the fibrotic changes and underlying liver disease observed in such cases.

According to our literature review, 24 cases of portosystemic encephalopathy without liver cirrhosis have been reported, including 20 from East Asia (3-15) and four from the USA (16-19), since Raskin et al. reported the first case in 1964 (16). Previous studies involved mostly women $(83 \%)$, with a mean age of 67.5 years (range: $37-86$ years). In previous reports, the portosystemic shunts were probably congenital; however, patients may be underdiagnosed simply because most physicians are unaware of this condition. Most reported cases of portosystemic encephalopathy caused by a spontaneous portosystemic shunt have occurred in subjects in their fifties, sixties and seventies. The reasons for this observation include the possibility that aging of the brain decreases the resistance of the brain tissue to the effects of ammonia and other metabolites (16), thus inducing damage to the liver parenchyma due to long-term ischemic changes associated with aging and a gradual increase in the blood flow in the shunt (17). In the present case, the following features led to the diagnosis of a spontaneous portosystemic shunt. The patient had no history of hepatic disease, and a physical examination did not reveal any signs of hepatic dysfunction. In addition, she had no history of abdominal surgery, and images showed no evidence of changes indicating liver cirrhosis or portal hypertension. Finally, serologic assays showed prior hepatitis B virus infection, and the patient exhibited no evidence of cirrhosis.

Among the cases identified in our literature review, the average time from the onset of the first symptom to diagno- sis was highly variable (range: 1 day-10 years). Many patients showed psychiatric and/or neurological symptoms, and four cases were misdiagnosed as dementia $(4,7,10,20)$. Notably, the patient in our case was misdiagnosed as having depression. Although none of the previous cases were misdiagnosed as depression, portosystemic encephalopathy caused symptoms similar to depression, such as subtle psychiatric and behavioral changes (21). Watanabe et al. (1) described characteristics suspicious of portosystemic encephalopathy in patients without liver cirrhosis: 1) high blood ammonia and bile acid levels with no or slight abnormalities in the liver function, 2) the repeated development of disturbance of psychiatric symptoms in psychiatric patients and 3) abnormally large blood vessels with no portal flow detected on abdominal imaging. These findings may provide useful clues for primary care physicians in addition to the variable symptoms of portosystemic encephalopathy. In the present case, there appeared to be two reasons for the late diagnosis. First, the patient had no history of liver disease and the findings of a physical examination were not consistent with liver cirrhosis. Second, the serum ammonia level was not routinely measured.

Although surgical intervention was previously the only treatment option for portosystemic shunts, interventional radiology techniques, such as B-RTO, now constitute a more favorable, less invasive option. Nevertheless, B-RTO is associated with two major complications: early procedure-related complications and late portal hypertensive complications. Procedure-related complications include infection, contrastinduced nephropathy, hematoma formation, pulmonary embolism due to the migration of coils or thrombi, hemolysis and pulmonary edema resulting from the use of ethanolamine oleate iopamidol $(22,23)$. Haptoglobin is usually administered to prevent critical hemolysis, which may cause nephropathy $(24,25)$, as in the present case. Portal hyper- 
tensive complications due to reperfusion of the shunt vessel flow include the de novo occurrence or aggravation of preexisting gastroesophageal varices or portal hypertensive gastropathy, ascites or spontaneous bacterial peritonitis $(22,23,26)$.

In a European multicenter cohort study, $41 \%$ of the patients with liver cirrhosis experienced recurrent hepatic encephalopathy within 100 days after embolization (26). However, in our literature review, recurrent hepatic encephalopathy was not reported in patients without liver cirrhosis, and all patients were free of hepatic encephalopathy for more than 12 months $(8-10,15,19)$. Embolization of portosystemic shunts may be more effective in patients without liver cirrhosis due to their greater preservation of the liver function and reduced portal blood pressure versus those with liver cirrhosis. In the present case, a notable improvement was observed in the patient's clinical status after successful treatment, and no signs of recurrent hepatic encephalopathy or portal hypertensive complications were reported after approximately eight months.

In conclusion, the possibility of a portosystemic shunt should be considered in the presence or absence of underlying liver disease and the ammonia level should be measured before diagnosing depression, as portosystemic encephalopathy may be reversible with interventional radiology treatment.

The authors state that they have no Conflict of Interest (COI).

\section{References}

1. Watanabe A. Portal-systemic encephalopathy in non-cirrhotic patients: classification of clinical types, diagnosis and treatment. J Gastroenterol Hepatol 15: 969-979, 2000.

2. Akahoshi T, Nishizaki T, Wakasugi K, et al. Portal-systemic encephalopathy due to a congenital extrahepatic portosystemic shunt: three cases and literature review. Hepatogastroenterology 47: 1113-1116, 2000

3. Mori H, Hayashi K, Fukuda T, et al. Intrahepatic portosystemic venous shunt: occurrence in patients with and without liver cirrhosis. Am J Roentgenol 149: 711-714, 1987.

4. Saijyo S, Kudo T, Suzuki M, et al. Establishment of a new extrahepatic bile duct carcinoma cell line, TFK-1. Tohoku J Exp Med 177: 61-71, 1995

5. Nishimoto Y, Hoshino H, Sato S, et al. Extrahepatic portosystemic venous shunt without portal hypertension. Intern Med 36: 886889, 1997.

6. Otake M, Kobayashi Y, Hashimoto D, et al. An inferior mesenteric-caval shunt via the internal iliac vein with portosystemic encephalopathy. Intern Med 40: 887-890, 2001.

7. KCS, Matsutani S, Maruyama H, et al. Portal-systemic encephalopathy in two patients without liver cirrhosis and portal hypertension. Hepatol Res 23: 122-129, 2002.

8. Tanaka O, Ishihara $\mathrm{K}$, Oyamada $\mathrm{H}$, et al. Successful portalsystemic shunt occlusion with balloon-occluded retrograde transvenous obliteration for portosystemic encephalopathy without liver cirrhosis. J Vasc Interv Radiol 17: 1951-1955, 2006.
9. Park SW, Kang HS, Kim YJ, Lee MW, Roh HG. Successful occlusion of spontaneous portosystemic shunts leading to encephalopathy in a non-cirrhotic patient by using the amplatzer vascular plug. Acta Radiol 48: 1077-1081, 2007.

10. Miyata K, Tamai H, Uno A, et al. Congenital portal systemic encephalopathy misdiagnosed as senile dementia. Intern Med 48: 321-324, 2009

11. Saito M, Seo Y, Yano Y, et al. Successful treatment using coil embolization of a symptomatic intrahepatic portosystemic venous shunt developing through a patent ductus venosus in a noncirrhotic adult. Intern Med 52: 555-559, 2013.

12. Takeuchi H, Takeda Y, Takahashi M, Hayashi S, Fukuzawa Y, Nakano T. A rare congenital extrahepatic portosystemic shunt affecting the inferior mesenteric vein, inferior vena cava, and left ovarian vein. Surg Radiol Anat 36: 729-732, 2014.

13. Yokota $T$, Tsuchiya $K$, Umetani $K$, Furukawa $T$, Tsukagoshi $H$. Choreoathetoid movements associated with a spleno-renal shunt. J Neurol 235: 487-488, 1988

14. Ohwada S, Hamada Y, Morishita Y, et al. Hepatic encephalopathy due to congenital splenorenal shunts: report of a case. Surg Today 24: 145-149, 1994.

15. Kuramitsu T, Komatsu M, Matsudaira N, et al. Portal-systemic encephalopathy from a spontaneous gastrorenal shunt diagnosed by three-dimensional computed tomography and treated effectively by percutaneous vascular embolization. Liver 18: 208-212, 1998.

16. Raskin NH, Price JB, Fishman RA. Portal-systemic encephalopathy due to congenital intrahepatic shunts. N Engl J Med 270: 225229, 1964.

17. Kerlan RK, Sollenberger RD, Palubinskas AJ, Raskin NH, Callen PW, Ehrenfeld WK. Portal-systemic encephalopathy due to a congenital portocaval shunt. AJR Am J Roentgenol 139: 1013-1015, 1982

18. Ali S, Stolpen AH, Schmidt WN. Portosystemic encephalopathy due to mesoiliac shunt in a patient without cirrhosis. J Clin Gastroenterol 44: 381-383, 2010.

19. Tsai CW, Kuo CC, Huang CC. Spontaneous, non-cirrhotic, intrahepatic porto-systemic shunt presenting as acute encephalopathy. Liver Int 34: 1603, 2014.

20. Kiriyama M, Takashima S, Sahara H, et al. Case report: portalsystemic encephalopathy due to a congenital extrahepatic portosystemic shunt. J Gastroenterol Hepatol 11: 626-629, 1996.

21. Albrecht J, Jones EA. Hepatic encephalopathy: molecular mechanisms underlying the clinical syndrome. J Neurol Sci 170: 138146, 1999.

22. Shimoda R, Horiuchi K, Hagiwara S, et al. Short-term complications of retrograde transvenous obliteration of gastric varices in patients with portal hypertension: effects of obliteration of major portosystemic shunts. Abdom Imaging 30: 306-313, 2005.

23. Cho SK, Shin SW, Lee IH, et al. Balloon-occluded retrograde transvenous obliteration of gastric varices: outcomes and complications in 49 patients. AJR Am J Roentgenol 189: W365-W372, 2007.

24. Schaer DJ, Buehler PW, Alayash AI, Belcher JD, Vercellotti GM. Hemolysis and free hemoglobin revisited: exploring hemoglobin and hemin scavengers as a novel class of therapeutic proteins. Blood 121: 1276-1284, 2013.

25. Hashizume M, Kitano S, Yamaga H, Sugimachi K. Haptoglobin to protect against renal damage from ethanolamine oleate sclerosant. Lancet 2: 340-341, 1988.

26. Laleman W, Simon-Talero M, Maleux G, et al. Embolization of large spontaneous portosystemic shunts for refractory hepatic encephalopathy: a multicenter survey on safety and efficacy. Hepatology 57: 2448-2457, 2013.

(C) 2015 The Japanese Society of Internal Medicine http://www.naika.or.jp/imonline/index.html 